\title{
Ueber den neueren Montebrasit von Descloizeaux (Hebronit);
}

von

Fr. V. Kobell.

(Aus den Sitzungsberiohten der königl. Bayer. Akad. d. Wissensch. zu Mübohen, Januar 1873, mitgetheilt vom Verf.)

Das Fluophosphat von Montebras, welches Descloizeaux auf Grund einer chemischen Analyse von Moissenet und auf Grund des optischen Verhaltens als eine vom ähnlichen Amblygonit verschiedene und eigenthümliehe Species erklärt hat ${ }^{1}$ ), ist von $\mathrm{Pi} \mathrm{sani}^{2}$ ), von $\mathrm{R}$ a m mels berg ${ }^{2}$ ) und von mir ${ }^{4}$ ) analysirt worden. Diese, ganz unabhängig von einander ausgeführten Analysen haben gezeigt, dass das fragliche Mineral keine neue Species, sondern Amblygonit sei. Descloizeaux hat das nun auch anerkannt und das Resultat seiner neuen optischen Untersuchung des Amblygonit von Penig hat sich als iibereinstimmend mit dem Verhalten des Minerals von Montebras erwiesen. Anders aber hat sich das Verhalten des Amblygonit von Hebron in Maine und eines neuerlich vorgekommeneu ähnlichen Minersls von Montebras gezeigt, welche von Pisani ${ }^{5}$ ) analysirt worden und unter anderv dureh einen Wassergehalt von 4 p. Ct. gegenüber dèm wasserfreien Amblygonit charakterisirt sind. Die Differenzen bestimmen nun Descloizeaux, auf das wasserhaltige Mineral den Namen Montebrasit zu übertragen, so dass zwischen älterem und neuerem Montebrasit zu unterscheiden ist. Ich habe von jeher dem Bestimmer einer neuen Species das Recht der

1) Mémoire sur un nouveau Fluophosphate trouvé dans le gite d'Etain de Montebras (Creuse) par M. L. Moissenet etc. avec une Note sur la Montebrasite par M. Des Cloizeaux, Paris 1871.

2) Comptes rendns 73, 1871.

s) Berichte der chemischein Gesellschaft in Berlin. 1872.

4) Sitzungsberichte cer Königl. Bayer. Akad. d. Wiss. 1872.

๖) Ann. ch. phys., 27, 1872. 
Taufe zugesprochen und anf die Nachtheile beliebter Umtaufeu hingewiesen, nachdem sich herausgestellt hat, dass Namen wie sie einer ideal einigermassen vollkommenen Nomenklatur entsprechen, allgernein nicht herstellbar sind; im vorliegenden Falle scheint mir aber doch unzulässig, wenn man, un nicht missverstanden zu werden, von einem iilteren und von einem neueren Montebrasit sprechen muss und ich schlage desshalb vor, den Namen Montebrasit, überhaupt fallen zu lassen und das wasserhaltige lluorphosphat von Hebron und ron Montebras mit dem Namen Hebron it za bezeichneu, inden ich gerne auf diesen Namen verzichte, wenn Descloizeaux selbst irgend einen andern statt Montebrasit für das von ihm bestimmte wasserhaltige Mineral zu geben geneigt ist. Linstweilen werde ich für letateres den Namen Hebronit gebrauchen.

Der Hebronit hat in der Krystallisation grosse Aehnlichkeit mit dem Amblygonit, sie ist nach Descloizeaux klinorhomboidiseh und zwei Spaltungsrichtungen bilden einen Winkel von $105^{\circ}$, während er beim Amblygonit $105^{\circ}$ $44^{\prime}$ ist. Ich habe einen Hebronit von Auburn in Maine untersucht und den Winkel annähernd zu $105 \%{ }^{\circ}$ gefunden, eine dritte Spaltuny'srichtung, die Descloizean $x$ angiebl, konote ich nicht deutlich erkennen.

Die Analyse Pisani's voun Hebronit von Hebron 1. und vom Hebronit von Montebras 2. gaben:

1.

Phosphorsiuure 46,65

Thonerde . . 36,00

lithium . . 4,56

Fluor . . 5,22

Wasser . $\cdot \frac{4,20}{90,60}$
2.

47,15

36,90

4,60

3,80

4,75

97,20

Die Proben haben den Schmelzgrarl des Amblygonit und färben die Löthrohrtlanme rein carminroth, die Varietät von Auburn fiabt aber die Nlanme rothgelb, so dass oflenbar der, wern auch geringe Gehalt an Natrium das Roth des Lithiums verïndert. Der Hebronit von Ankurn 
nud ebenso der von Paris in Maine phosphoresciren erwärmt ziemlich stark mit bläulichem Lichte.

Das spec. Gewicht des Hebronit von Auburn ist 3,06. Die Phosphorsäure ist im Amblygonit wie in Hebronit leicht nachzuweisen, da sich bei kurzem Kochen des feinen Pulvers ınit Salpetersäure hinlünglich viel anflüst, um mit molybdänsanrem Ammoniak das gelbe Priicipitat za geben.

leh habe die Analyse des Hebronit von Auburn in derselben Weise durehgefiilurt wie ich bei der Analyse des A unblygonit von Montebras beschirieben. Das Resultat war:

Phosphorsänre 49,00

Thounde . . 37,00

Lithium . 3,44

Natrium . . 0,79

Fluor . . 5,50

Wasser . . 4,50

$$
100,23
$$

Vergleicht man diese Mischung wit der des Amblygonit, so zeigt sich, dass die Differenzen wesentlich die Fluoride und den Wassergehalt betreften, wïhrend das Thonerdephosphat in beiden Species dassebe ist. Rammelsberg hat für den Amblygonit hie fiormel gegrebeu

$$
\left\{\begin{array}{l}
2 \mathrm{AlP}_{2} \mathrm{O}_{8} \\
3 \mathrm{RF}
\end{array}\right\} \text { d. i. } 2 \dddot{\mathrm{A}}(\ddot{\mathrm{P}}+3 \mathrm{BFl} \text { ) }
$$

In der Varietät von Penig ist $\mathrm{R}$ dureh $\% / 6$ Li und $\% / 5 \mathrm{Na}$ vertrecen and so wird die speciellere Formel dafiur

wounch sich berechnet:

$$
3 \dddot{A} 1 \ddot{\mathrm{P}}+3\left\{\begin{array}{ll}
1 / 5 & \mathrm{Li} \\
1 / 5 & \mathrm{Na}
\end{array}\right\} \mathrm{Fl}
$$

\section{Phosphorsäure 49,17}

'Thonerde . . 35,67

Lithium . 2,91

Natrium . 2, 2,39

Fluor . . 9,86

$$
100,00
$$

1) $\mathrm{O}=16, \mathrm{Li}:=7, \quad \mathrm{l}=19$; fịr Al findet man in den neueren Augaben 27,$3 ; 27,4 ; 27,6$; Al ist bei $R$ a m melsberg 54,6 , in Strecker's Jahresbericht für 1869 ist $\mathrm{Al}=2 \mathrm{~T}, 4$. 
Meine Analyse des Amblygonit von Montebras führt annähernd zu der ähnlichen Formel

$$
2(\overline{\mathrm{Al}} \ddot{\overline{\mathbf{P}}})+3\left\{\begin{array}{ll}
5 / 7 & \mathrm{Li} \\
2 / 7 & \mathrm{Na}
\end{array}\right\} \mathrm{Fl}
$$

Für den Hebronit von Auburn kann man die Formel schreiben

$$
3(\dddot{\mathrm{A}} \mathrm{l} \ddot{\mathrm{P}})+\mathrm{Li}_{5} \mathrm{Fl}_{2}+2 \mathrm{H}_{2}
$$

Sie giebt (das wenige Natrium in Lithium übersetzt):

$$
\begin{aligned}
& \text { Phosphorsäure 50,36 } \\
& \text { Thonerde . } 36,52 \\
& \text { Lithium . . 4,37 } \\
& \text { Fluor . - 4,50 } \\
& \text { Wasser . . } 4,25 \\
& 100,00
\end{aligned}
$$

Da die Analysen des Hebronit von Hebron, von Montebras und von Auburn den Wassergehalt ijbereinstimmend geben, so kann man diesen Gehalt nicht als zufällig ansehen und obwohl der Verlust der Analysen Pisani's grösser ist als gewöhnlich vorkommt, so zeigt sich doch, dass der Fluorgehalt des Hebronit merklich geringer als beim Amblygonit nnd sich, auch wenn der Hebronit als wasserfrei berechnet wird, wie $5: 9$ verhält. Die Differenz des Spaltungswinkels beider Mineralien beträgt zwar nach Descloizeaux, welchem gat spiegelnde Stricke zu Gebot standen, nur 44', das optische Vorhalten ist aber nach seinen ausfïhrlichen Untersuchungen in der Art verschieden, dass heim Amblygonit die Dispersion der optischen Axen fïr die rothen Strahlen grösser ist als für die violetten, während es aich beim Hebronit umgekehrt verhält. Descloizeaux sagt dariber: "cette opposition dans la dispersion propre des axes optiques suffirait pour séparer l'amblygonite de la montebrasite (unser Hebronit), car elle constitue un caractère distinctif de la plus hante importance dans les substances erystallisées, et elle parait être la plns constante de tous leurs propriétés optiques birefringentes. 
v. Kobell: Ueber den neveren Montebrasit. 49

Il est, en effet, sans exemple aue des echantillons d'uno même espèce bien définie, naturelle ou artificielle, quelles que soit d'ailleurs les variations de leurs caracteres physiques ou chimiques, possèdent des axes optiques a dispersions opposées, tant que ses axes restent situes dans !e même plan etc.

Wenn der Hebronit das Produkt einer anfangenden Zersetzung des Amblygonit wäre, wie nach dem geringeren Fluorgehalt und dem Zutreten des Wassers geschlossen werden könnte, so wäre er ein Gemenge von Amblygonit und einem lithionhaltigen Thonphosphat mit Wasser. Um darüber năheren Aufschluss zn erhalten, kann man mit den 5,5 pr. Ct. Fluor die Menge des noch darin enthaltenen Amblygonit berechnen, die sich zu 54,85 ergiebt.

Diese enthalten:

Phosphorsänre 27,43

Thonerde . . 19,90

Lithium . 2,02

Fluor . . 5,50

$$
54,85
$$

Nach Abzug dieses Antheils von der Mischung des Hebronit bleiben:

Phosphorsäure 21,57

Thonerde . . 17,10

Lithion . . 3,55 (die 1,66 Li als Li)

Wasser . . 4,60

46,72 Hebronit.

Aus dieser Mischung kann die Formel gebildet werden:

welche ergiebt:

$$
4(\ddot{A l})+3 \text { छï̈ }_{2}
$$

$$
\begin{aligned}
& \text { Phosphorsänre } 22,53 \\
& \text { Thonerde . } \quad 16,84 \\
& \text { Lithion . . } \quad 3,56 \\
& \text { Wasser . . } \frac{4,29}{46,72}
\end{aligned}
$$

Weniger einfach wiinde die Formel mit Herstellung eines Lithionphosphats. Fin Gemenge aber, wie das hier 
angedeutete, würde den optischen Charakter nicht wohl zeigen können, wie ihn Descloi zea ux angegeben, und somit scheint mir, dase zur 'Keit das besprochene Mineral, walches auch sonst nicht wie ein in Zersetyung begriffenes asussieht, als eine eigenthümliche Species aufzunehmen sei. Der Hebronit von Hebron und von Auburn ist von rötblichew Lithionit begleitet.

\title{
Zur Frage über die Einführung der modernen chernischen Formeln in die Mineralogie.
}

\author{
von

\section{v. K.obell.}

(Aus den Sitzungsberichteu der königl. Bayer. Akad. d. Wissensoh. zu München, Februar 1873, mitgetheilt vom Verf.)

Wenu man in den chemischen Formeln nur die verbundenen Elemente angiebt und die Anzahl ihrer Atome, so lässt man die Frage, wie sie zu näheren Verbindungen geeinigt seien, offen; went man die nächlet näheren Verbiadungen berücksichtigt, so liefert man das Material zu einer rationellen Formel, welche verschieden construirt werden kann, je nach den Gesichtspunkten, von denen man ausgeht, und je nach den Kwecken, welchen eine solche Formel dienen soll. Dass daher, besonders für complicirte Mischungen, die verschiedensten F'ormeln aufgestellt werden können, ist selbstverständlich und ebenso, dass die des Theoretikers und die des Praktikers in der Chemie sehr verschieden sein können. Natürlich müssen sie sich alle durch Rechnung in einander verwandeln und auf die Resultate der Analyse, welche sie beleuchten sollen, zurückführen lassen. Die Anhänger der sog. modernen Chemie wollev die bisherigen Formeln des Systems von Berzelius nicht mehr gelten lassen und die Mineralogie soll ihre atomistisch - empiribchen oder weiter gehend ihre zu theoretischen Betrachtangen und Speculationen entworfenen 